\title{
A COHOMOLOGICAL TAMAGAWA NUMBER FORMULA
}

\author{
ANNETTE HUBER AND GUIDO KINGS
}

\begin{abstract}
For smooth linear group schemes over $\mathbb{Z}$, we give a cohomological interpretation of the local Tamagawa measures as cohomological periods. This is in the spirit of the Tamagawa measures for motives defined by Bloch and Kato. We show that in the case of tori, the cohomological and the motivic Tamagawa measures coincide, which proves again the Bloch-Kato conjecture for motives associated to tori.
\end{abstract}

\section{Introduction}

The purpose of this article is twofold: we propose and justify a new definition of $p$-adic periods for reductive groups, and we give evidence that it is related to the Tamagawa number conjecture of Bloch and Kato.

Recall that the complex period numbers of an algebraic variety $X / \mathbb{Q}$ are defined as the values of the natural pairing (given by integration) between algebraic de Rham cohomology over $\mathbb{Q}$ and rational singular homology of $X(\mathbb{C})$. Note that this pairing can also be phrased as an isomorphism between de Rham cohomology and singular cohomology. In the case of reductive groups, we want to imitate this in the setting of $p$-adic analysis. We use the following dictionary: de Rham cohomology is replaced by Lie algebra cohomology; singular cohomology is replaced by analytic group cohomology; the comparison isomorphism is the one of Lazard (see Section 1.3 for details). For every model $G / \mathbb{Z}$ of a reductive group and a Lie algebra class $[\omega]$ of top degree, this defines a period number $\mu_{[\omega]}^{\mathrm{coh}}\left(G\left(\mathbb{Z}_{p}\right)\right)$ (see Definition 1.3.5). In the first part of the article, we prove that the product of these cohomological periods is related to values of $L$-functions. The result is particularly easy to state in the case of $\mathrm{Sl}_{n}$.

Received August 17, 2009. Revised June 9, 2010. Accepted August 10, 2010.

2010 Mathematics Subject Classification. 11G40, 11R42, 14G10, 22E41.

(C) 2011 by The Editorial Board of the Nagoya Mathematical Journal 
Theorem 1. Let $[\omega] \in H^{n^{2}-1}\left(\operatorname{Lie}\left(\mathrm{Sl}_{n}\right), \mathbb{Z}\right)$ be a basis element. Then

$$
\prod_{p} \mu_{[\omega]}^{\mathrm{coh}}\left(\mathrm{Sl}_{n}\left(\mathbb{Z}_{p}\right)\right)=[\zeta(2) \zeta(3) \cdots \zeta(n)]^{-1},
$$

where $\zeta(s)$ is the Riemann $\zeta$-function.

For general reductive groups, this takes the form of a cohomological Tamagawa number formula taking also the infinite place into account (see Theorem 1.5.7). The key ingredient is an integral version of Lazard's isomorphism proved in [HKN].

The Tamagawa number formula for a reductive algebraic group $G$ relates the volume of $G(\mathbb{A}) / G(\mathbb{Q})$ to arithmetical invariants of $G$. Bloch and Kato proposed a similar formula for all motives, which unfortunately remains largely conjectural. They define $p$-adic periods by comparing different $p$ adic cohomology theories via the Bloch-Kato exponential map. There is no direct link to the classical Tamagawa measure for linear groups. At the end of the introduction of $[\mathrm{BK}]$, Bloch and Kato state that they see the intersection between the theory of algebraic groups and the theory of motives as consisting of abelian varieties and tori. Indeed, the very formulation of the Bloch-Kato conjecture is inspired by Bloch's beautiful paper [B], where he shows that the conjecture of Birch and Swinnerton-Dyer is equivalent to a Tamagawa number formula in the sense of algebraic groups.

It has been our philosophy to also view reductive groups in this intersection. We see the Lazard isomorphism as the $p$-adic part of the bridge. There is some evidence for such a connection. In the case of the algebraic group for a division algebra (see Example 1.1.8), the infinite cohomological period is very closely related to Borel's regulator in [Bo]. The work of Borel, complemented by a comparison of regulators due to Beilinson and Rapoport, provides this relation for the "infinite period," proving the Beilinson conjecture, that is, the Bloch-Kato conjecture up to a rational factor. On the other hand, we have shown in [HK2] that the $p$-adic cohomological periods are related to the Bloch-Kato exponential map and the Soulé regulator, which are used in the definition of the local motivic Tamagawa measures. This article proves that our cohomological periods are the right choice to correct Borel's rational formula. (Unfortunately, it is by no means obvious how to prove the conjecture from these ingredients.)

As pointed out by a referee, the cohomological Tamagawa number formula of this paper could probably be generalized to more general algebraic group 
schemes, and by [B] even a relation to the Bloch-Kato conjecture is possible. However, we restrict to the reductive case because [Bo] and [HK2] are strictly restricted to the setting of reductive groups.

In the second part of this article, we treat the case of tori as a test case for our approach. We show that our cohomological Tamagawa measures can be identified with the motivic Tamagawa measures of Bloch and Kato. Together with the key input of Ono's classical Tamagawa number formula for tori (see $[\mathrm{O}]$ ), this gives a proof of the Tamagawa number formula for the motives associated to tori over $\mathbb{Q}$. The result as such is not new (see $[\mathrm{F}$, section 8.3]). By general yoga for Artin motives over $\mathbb{Q}$, it suffices to treat the case of $h^{0}(F)$ where $F$ is a number field, that is, the Dedekind $\zeta$-function at zero (resp., 1). This is nothing but the class number formula. However, the direct proof is another piece of evidence for our approach to the Bloch-Kato conjecture for reductive groups, or, equivalently, for Dedekind $\zeta$-functions.

\section{$\S 1$. A cohomological Tamagawa measure}

\subsection{Notation}

We fix the following setting for the whole article.

Let $G$ be a smooth linear group scheme over $\mathbb{Z}$ whose generic fiber is connected reductive. If $R$ is a ring, we denote by $G(R)$ the $R$-valued points of $G$, and we denote by $G_{R}$ the base change to $R$.

For any finite place $p$, we choose $G_{p}^{c}$, a compact open subgroup of $G\left(\mathbb{Q}_{p}\right)$.

We say that the pair $\left(G, G_{p}^{c}\right)$ with $(p<\infty)$ has good reduction at $p$ if $G_{p}^{c}=G\left(\mathbb{Z}_{p}\right)$ and $G_{\mathbb{F}_{p}}$ is quasi-split reductive. In particular, $G_{c}^{p}$ is a maximal compact at good reduction primes $p$.

We assume that $\left(G, G_{p}^{c}\right)$ has good reduction at almost all places.

For $p=\infty$, we put $G_{\infty}^{c}=G(\mathbb{R})$ (which is, of course, not compact in general).

REMARK 1.1.1. We could choose $G_{p}^{c}=G\left(\mathbb{Z}_{p}\right)$ for all finite places $p$. Recall also that by $\left[\mathrm{PR}\right.$, Proposition 3.16], any compact group of $G\left(\mathbb{Q}_{p}\right)$ is contained in a maximal compact subgroup.

Let $\mathbb{A}$ be the ring of adeles of $\mathbb{Q}$, and let $\mathbb{A}_{f}$ be the ring of finite adeles. We put

$$
K_{f}:=\prod_{p<\infty} G_{p}^{c} \subset G\left(\mathbb{A}_{f}\right) .
$$

It is a compact open subgroup of $G\left(\mathbb{A}_{f}\right)$ and of finite index in a maximal compact open subgroup. As usual, we embed $G(\mathbb{Q})$ diagonally into $G(\mathbb{A})$. 
We denote by

$$
H_{G, K_{f}}=G(\mathbb{Q}) \backslash G(\mathbb{A}) / G(\mathbb{R}) K_{f}
$$

the set of double cosets. For all $g \in G(\mathbb{A})$, let

$$
\Gamma_{g}=g G(\mathbb{R}) K_{f} g^{-1} \cap G(\mathbb{Q}) \subset G(\mathbb{R}) .
$$

In particular,

$$
\Gamma:=\Gamma_{e}=\left\{g \in G(\mathbb{Q}) \mid g \in G_{p}^{c} \text { for all finite places } p\right\} .
$$

LEMMA 1.1.2. We have the following:

(1) $H_{G, K_{f}}$ is finite;

(2) for all $g \in G(\mathbb{A})$, the group $\Gamma_{g}$ is an arithmetic subgroup of $G(\mathbb{R})$, that depends only on the class of $g$ in $H_{G, K_{f}}$;

$$
G(\mathbb{Q}) \backslash G(\mathbb{A}) / K_{f}=\coprod_{g \in H_{G, K_{f}}} \Gamma_{g} \backslash G(\mathbb{R}) .
$$

Proof. As $K_{f}$ is of finite index in a maximal compact subgroup, the first statement follows from [PR, Theorem 5.1]. Independence of the representative of the class is easy to check. The last statement is a standard computation: for $x, x^{\prime} \in G(\mathbb{R})$, the double cosets $G(\mathbb{Q}) g x K_{f}$ and $G(\mathbb{Q}) g x^{\prime} K_{f}$ are equal if and only if there exists a $\gamma \in G(\mathbb{Q})$ with $\gamma g x K_{f}=g x^{\prime} K_{f}$. This is equivalent to $\gamma \in g G(\mathbb{R}) K_{f} g^{-1}$ and $\gamma x=x^{\prime}$. Thus, the double classes of $G(\mathbb{Q}) g G(\mathbb{R}) K_{f}$ are represented by $\Gamma_{g} \backslash G(\mathbb{R})$.

Definition 1.1.3. We call the number of double cosets

$$
h_{G, K_{f}}=\# H_{G, K_{f}}
$$

the class number of $G$ relative to $K_{f}$.

EXAMPLE 1.1.4. Let $K$ be a number field with ring of integers $\mathcal{O}$, and consider the Weil restriction $G=\operatorname{Res}_{\mathcal{O} / \mathbb{Z}} \mathbb{G}_{m}$ with $G_{p}^{c}=G\left(\mathbb{Z}_{p}\right)=\left(\mathcal{O} \otimes \mathbb{Z}_{p}\right)^{*}$ for all primes $p$. Then $h_{G, K_{f}}$ is the class number of $K$. In this case, all $\Gamma_{g}$ are equal to $\Gamma$.

ExAmple 1.1.5. Assume that $G_{\mathbb{Q}}$ is simple, simply connected with $G(\mathbb{R})$ noncompact. Then by the strong approximation theorem (see [PR, Theorem 7.12$]$ ), $h_{G, K_{f}}=1$ (for any choice of $K_{f} !$ ). Hence,

$$
G(\mathbb{Q}) \backslash G(\mathbb{A}) / K_{f}=\Gamma \backslash G(\mathbb{R}) .
$$


We need a technical condition.

Assumption 1.1.6. We assume that $G(\mathbb{A}) / G(\mathbb{Q})$ is compact (or, equivalently, that all $\Gamma_{g} \backslash G(\mathbb{R})$ are compact).

Note that the condition depends only on $G_{\mathbb{Q}}$. In fact, by $[\mathrm{PR}$, Theorem 4.12], it is equivalent to $G_{\mathbb{Q}}$ being anisotropic.

EXAMPLE 1.1.7. Let $T$ be a smooth group scheme over $\mathbb{Z}$ whose generic fiber is a torus. Then the assumption is equivalent to the condition that the $\mathbb{Q}$-rank of $T$ is zero (see $\left[\mathrm{PR}\right.$, Theorem 4.11]). Hence, the torus $\operatorname{Res}_{\mathcal{O} / \mathbb{Z}} \mathbb{G}_{m}$ does not satisfy the assumption, but the quotient $\operatorname{Res}_{\mathcal{O} / \mathbb{Z}} \mathbb{G}_{m} / \mathbb{G}_{m}$ does. We consider this example in more detail in the second part of the article.

ExAmPle 1.1.8. Let $D$ be a noncommutative division algebra over $\mathbb{Q}$ with center $F$. Let $\mathcal{D}$ be a maximal $\mathbb{Z}$-order of $D$. (They exist; see, e.g., [CR, Corollary 26.6].) Let $G$ be the group scheme of units of a $\mathcal{D}$, that is, given by the functor

$$
G(A)=(\mathcal{D} \otimes A)^{*}
$$

for all rings $A$. The group $G$ is easily seen to be smooth. $G_{\mathbb{Q}}$ is reductive. However, it does not satisfy our assumption because it contains a diagonal torus $\mathbb{G}_{m}$. Let $H$ be the kernel of the reduced norm map. More precisely, for all commutative $\mathcal{O}_{F}$-algebras $A^{\prime}$, consider

$$
\text { nred : }\left(\mathcal{D} \otimes \mathcal{O}_{F} A^{\prime}\right)^{*} \rightarrow\left(A^{\prime}\right)^{*}
$$

(see [CR, Section 7D, Corollary 26.2]). Let $H^{\prime}$ be the algebraic group over $\mathcal{O}_{F}$ defined by its kernel. It is a form of $\mathrm{Sl}_{d} / F$, in particular simple, and we let

$$
H=\operatorname{Res}_{\mathcal{O}_{F} / \mathbb{Z}} H^{\prime}
$$

Then $H_{\mathbb{Q}}$ is a form of $\operatorname{Res}_{F / \mathbb{Q}} \mathrm{Sl}_{d}$, in particular semisimple and simply connected. The group is anisotropic, and hence it satisfies Assumption 1.1.6. The smoothness of $H / \mathbb{Z}$ is not obvious. For a direct proof, see [N, Theorem 2].

The invariant $h_{G}$ for $H$ is 1 if strong approximation holds for $H^{\prime}$, that is, if and only if $H^{\prime}\left(F_{v}\right)$ is noncompact for all infinite places $v$ of $F$. This is the case if the algebra $A^{\prime}$ is split at the infinite places but not true in general. 


\subsection{The local measure: real case}

Let $\omega$ be an invariant $d$-form on $G(\mathbb{R})$. Let $\Gamma_{g}$ be as in Section 1.1. Depending on the choice of $\omega$, we are going to introduce a measure on $\Gamma_{g} \backslash G(\mathbb{R})$. Recall that this quotient was assumed to be compact. Note that $\Gamma_{g} \backslash G(\mathbb{R})$ is not a manifold in general. It may have orbifold singularities.

Note also that $\Gamma_{g} \backslash G(\mathbb{R})$ is not connected in general. In order to simplify notation, we will assume that $G(\mathbb{R})$ is connected for the rest of this section. It is easy to extend the formulas to the general case.

Let $\mathfrak{g}$ be the algebraic Lie algebra of $G_{\mathbb{R}}$. It can be identified with the Lie algebra of the real Lie group $G(\mathbb{R})$.

The cohomological measure. Let $A_{G(\mathbb{R})}^{i}$ be the space of real $C_{\infty}$-differential forms on $G(\mathbb{R})$. By definition, $\mathfrak{g}^{\vee}=\left(A_{G(\mathbb{R})}^{1}\right)^{G(\mathbb{R})}$, the space of invariant oneforms. Moreover, choose $\Gamma^{\prime} \subset \Gamma_{g}$, a normal subgroup of finite index such that $\Gamma^{\prime} \backslash G(\mathbb{R})$ is a manifold. Together with Stokes's theorem, this defines natural maps

$$
\begin{aligned}
\grave{\Lambda} \mathfrak{g}^{\vee} & \rightarrow\left(A_{G(\mathbb{R})}^{\bullet}\right)^{G(\mathbb{R})} \subset\left(A_{G(\mathbb{R})}^{\bullet}\right)^{\Gamma_{g}} \\
& \rightarrow\left(A_{\Gamma^{\prime} \backslash G(\mathbb{R})}^{\bullet}\right)^{\Gamma_{g} / \Gamma^{\prime}} \rightarrow C_{\text {sing }}^{\bullet}\left(\Gamma^{\prime} \backslash G(\mathbb{R}), \mathbb{R}\right)^{\Gamma_{g} / \Gamma^{\prime}} \rightarrow C_{\text {sing }}^{\bullet}\left(\Gamma_{g} \backslash G(\mathbb{R}), \mathbb{R}\right) .
\end{aligned}
$$

The composition is independent of the choice of $\Gamma^{\prime}$.

DeFinition 1.2.1. Let

$$
\mathcal{S}: H^{i}(\mathfrak{g}, \mathbb{R}) \rightarrow H_{\text {sing }}^{i}\left(\Gamma_{g} \backslash G(\mathbb{R}), \mathbb{R}\right)
$$

be the natural map defined by Stokes's theorem, that is, the natural homomorphism induced by the above composition.

REMARK 1.2.2. Let $K_{\infty}$ be a maximal compact subgroup of $G(\mathbb{R})$ with Lie algebra $\mathfrak{k}$. Recall that the van Est isomorphism identifies relative Lie algebra cohomology with continuous group cohomology

$$
H^{i}(\mathfrak{g}, \mathfrak{k}, \mathbb{R}) \cong H_{\text {cont }}^{i}(G(\mathbb{R}), \mathbb{R}) .
$$

Restricting to $\Gamma_{g} \subset G(\mathbb{R})$ and identifying the cohomology of $\Gamma_{g}$ with $H_{\text {sing }}\left(\left(\Gamma_{g} \backslash G(\mathbb{R}) / K_{\infty}, \mathbb{R}\right)\right.$, we get a map

$$
H^{i}(\mathfrak{g}, \mathfrak{k}, \mathbb{R}) \rightarrow H_{\text {sing }}^{i}\left(\Gamma_{g} \backslash G(\mathbb{R}) / K_{\infty}, \mathbb{R}\right) .
$$

This map is a crucial ingredient in Borel's definition of a regulator for number fields. 
Definition 1.2.3. Let $\eta_{\Gamma_{g}} \in H^{d}\left(\Gamma_{g} \backslash G(\mathbb{R}), \mathbb{Q}\right)$ be the fundamental class, that is, the Poincaré dual of the cycle $\Gamma_{g} \backslash G(\mathbb{R})$ in $H^{0}\left(\Gamma_{g} \backslash G(\mathbb{R}), \mathbb{Q}\right)$.

The fundamental class is a basis of $H^{d}\left(\Gamma_{g} \backslash G(\mathbb{R}), \mathbb{Q}\right)$.

REMARK 1.2.4. If $G(\mathbb{R})$ is not connected, this construction has to be carried out in such a way that Lemma 1.2.6 holds. We leave the details to the reader.

Definition 1.2.5. Let $[\omega] \in H^{d}(\mathfrak{g}, \mathbb{R})$. Then

$$
\mathcal{S}([\omega])=\pi\left(\Gamma_{g},[\omega]\right) \eta_{\Gamma_{g}}
$$

for some real number, the cohomological period at infinity. Let $\mu_{[\omega]}^{\mathrm{coh}}$ be the unique translation-invariant measure on $\coprod_{g \in H_{G, K_{f}}} \Gamma_{g} \backslash G(\mathbb{R})$ normalized by

$$
\mu_{[\omega]}^{\mathrm{coh}}\left(\Gamma_{g} \backslash G(\mathbb{R})\right)=\pi\left(\Gamma_{g},[\omega]\right) .
$$

Lemma 1.2.6. Let $\Gamma^{\prime} \subset \Gamma_{g}$ be a normal subgroup of finite index. Then

$$
\pi\left(\Gamma^{\prime},[\omega]\right)=\left[\Gamma_{g}: \Gamma^{\prime}\right] \pi\left(\Gamma_{g},[\omega]\right)
$$

Proof. This follows directly from the projection formula for the covering $\Gamma^{\prime} \backslash G(\mathbb{R}) \rightarrow \Gamma_{g} \backslash G(\mathbb{R})$.

Let $\omega \neq 0$ be an invariant $d$-form on $G(\mathbb{R})$. It is a gauge form and hence defines a (nonnormalized) translation-invariant measure $\mu_{\omega}^{\text {Tam }}$ on the compact homogeneous space $\Gamma_{g} \backslash G(\mathbb{R})$. This is nothing but the local Tamagawa measure at infinity.

On the other hand, $\omega$ induces a class $[\omega] \in H^{d}(\mathfrak{g}, \mathbb{R})$.

Proposition 1.2.7. Let $\omega \neq 0$ be an invariant $d$-form on $G(\mathbb{R})$. Then

$$
\mu_{\omega}^{\mathrm{Tam}}=\mu_{[\omega]}^{\mathrm{coh}}
$$

The cohomological period agrees with the local Tamagawa number.

Proof. By Lemma 1.2.6, we may replace $\Gamma_{g}$ by a normal subgroup of finite index. We choose $\Gamma^{\prime}$ small enough such that $\Gamma^{\prime} \backslash G(\mathbb{R})$ is a manifold. By definition, the isomorphism $\mathcal{S}$ is induced by Stokes's theorem for $\Gamma^{\prime} \backslash G(\mathbb{R})$, that is, by integrating differential forms over cycles. 


\subsection{The local measure: $p$-adic case}

Let $G$ be a smooth algebraic group scheme over $\mathbb{Z}_{p}$ of dimension $d$ with connected generic fiber. Let $\mathfrak{g}$ be its $\mathbb{Z}_{p}$-Lie algebra. Let $\omega$ be an invariant $d$-form on $G_{\mathbb{Q}_{p}}$. Depending on the choice of $\omega$, we are going to introduce a measure on the compact group $G\left(\mathbb{Z}_{p}\right)$ and compare it with the classical local Tamagawa measure.

Recall that $G\left(\mathbb{Z}_{p}\right)$ is a $p$-adic Lie group. Let $\mathcal{G} \subset G\left(\mathbb{Z}_{p}\right)$ be a compact open subgroup. Recall also (see [L, chapitre V, théorème 2.3.10]) that continuous and locally analytic group cohomology agree for $\mathcal{G}$. We are going to denote it simply by $H^{i}\left(\mathcal{G}, \mathbb{Q}_{p}\right)$.

Proposition 1.3.1. The natural restriction map

$$
\text { res : } H^{i}\left(G\left(\mathbb{Z}_{p}\right), \mathbb{Q}_{p}\right) \rightarrow H^{i}\left(\mathcal{G}, \mathbb{Q}_{p}\right)
$$

is an isomorphism.

Proof. In [HKN, Theorem 4.3.1], locally analytic group cohomology of both groups was shown to agree with Lie algebra cohomology. In particular, the restriction is an isomorphism.

However, the integral structures on these cohomology groups defined by cohomology with coefficients in $\mathbb{Z}_{p}$ differ.

If $\mathcal{G}$ is without $p$-torsion, then by [L, chapitre $\mathrm{V}$, théorème 2.5.8], it is a Poincaré group with dualizing module $D=\mathbb{Z}_{p}$. This means that for every finitely generated $\mathbb{Z}_{p}$-module $A$ with continuous operation of $\mathcal{G}$, there is a natural isomorphism

$$
H^{i}\left(\mathcal{G}, A^{*}\right)^{*} \cong H^{d-i}(\mathcal{G}, A)
$$

(Here $(\cdot)^{*}$ is the Pontrjagin dual $\operatorname{Hom}\left(\cdot, \mathbb{Q}_{p} / \mathbb{Z}_{p}\right)$.) We are interested in the basic case $A=\mathbb{Z}_{p}$.

DEFINITION 1.3.2. Let $\mathcal{G} \subset G\left(\mathbb{Z}_{p}\right)$ be an open subgroup without torsion. We put $\eta_{\mathcal{G}} \in H^{d}\left(\mathcal{G}, \mathbb{Q}_{p}\right)$, the image of $1 \in \mathbb{Z}_{p}=H^{0}\left(\mathcal{G}, \mathbb{Q}_{p} / \mathbb{Z}_{p}\right)^{*}$, in $H^{d}\left(\mathcal{G}, \mathbb{Z}_{p}\right) \subset H^{d}\left(\mathcal{G}, \mathbb{Q}_{p}\right)$. It is called the fundamental class of $\mathcal{G}$.

Note that the fundamental class is a generator of $H^{d}\left(\mathcal{G}, \mathbb{Z}_{p}\right)$ as $\mathbb{Z}_{p}$-module. This condition determines it up to a factor in $\mathbb{Z}_{p}^{*}$.

REMARK 1.3.3. There is an alternative point of view. If $\mathcal{G}$ is a Poincaré group with dualizing module $D=\mathbb{Z}_{p}$, then there is also a natural $\mathbb{Q}_{p}$-duality. Under the perfect pairing

$$
H^{0}\left(\mathcal{G}, \mathbb{Q}_{p}\right) \times H^{d}\left(\mathcal{G}, \mathbb{Q}_{p}\right) \rightarrow \mathbb{Q}_{p}
$$


$\eta_{\mathcal{G}}$ is indeed the dual of $1 \in H^{0}\left(G, \mathbb{Q}_{p}\right)$. Details can be found elsewhere (see $[\mathrm{H}])$.

LEMMA 1.3.4. Let $\mathcal{G}$ be an open torsion-free subgroup of $G\left(\mathbb{Z}_{p}\right)$, and let $\mathcal{G}^{\prime} \subset \mathcal{G}$ be a subgroup of index $N=p^{k}$. Then

$$
\left.\eta_{\mathcal{G}}\right|_{\mathcal{G}^{\prime}}=p^{k} \eta_{\mathcal{G}^{\prime}}
$$

Proof. This follows from the projection formula for restriction and corestriction.

Let $[\omega] \in H^{d}\left(\mathfrak{g}, \mathbb{Q}_{p}\right)$. We think of it as a volume form on $G\left(\mathbb{Z}_{p}\right)$. It is represented by some invariant $d$-form $\omega$ on $G\left(\mathbb{Z}_{p}\right)$. Recall (see $[\mathrm{L}$, chapitre V, théorème 2.4.9] and [HKN, Theorem 4.3.1]) that the Lazard morphism is an isomorphism

$$
\operatorname{Laz}: H^{d}\left(\mathcal{G}, \mathbb{Q}_{p}\right) \rightarrow H^{d}\left(\mathfrak{g}, \mathbb{Q}_{p}\right) .
$$

Hence, $\operatorname{Laz}^{-1}[\omega]$ is a multiple of the fundamental class of $G\left(\mathbb{Z}_{p}\right)$. We define $\pi(\mathcal{G},[\omega]) \in \mathbb{Q}_{p}$ by

$$
\mathrm{Laz}^{-1}[\omega]=\pi(\mathcal{G},[\omega]) \eta_{\mathcal{G}}
$$

Definition 1.3.5. Let $\mathcal{G} \subset G\left(\mathbb{Z}_{p}\right)$ be an open subgroup, and let $[\omega] \in$ $H^{d}\left(\mathfrak{g}, \mathbb{Q}_{p}\right)$ be a cohomology class. Choose $\mathcal{G}^{\prime} \subset \mathcal{G}$, a torsion-free open subgroup. Let $\mu_{[\omega]}^{\mathrm{coh}}$ be the unique Haar measure on $\mathcal{G}$ normalized by

$$
\mu_{[\omega]}^{\mathrm{coh}}\left(\mathcal{G}^{\prime}\right)=\left|\pi\left(\mathcal{G}^{\prime},[\omega]\right)\right|_{p}
$$

LEMma 1.3.6. The cohomological measure is well defined, that is, independent of the choice of $\mathcal{G}^{\prime}$.

Proof. This follows directly from Lemma 1.3.4.

\subsection{The local Tamagawa measure}

An invariant $d$-form $\omega$ defines a local Tamagawa measure $\mu_{\omega}^{\text {Tam }}$ on $G_{p}^{c}$ (see [W, Section 2.2.1]). More precisely, let $U \subset G_{p}^{c}$, let $x_{1}, \ldots, x_{d}$ be local coordinates on $U$, and let $\omega=f d x_{1} \wedge d x_{2} \wedge \cdots \wedge d x_{d}$ on $U$. Then

$$
\mu_{\omega}^{\operatorname{Tam}}(U)=\int_{x(U)}\left|f \circ x^{-1}\right|_{p}
$$

with respect to the standard measure on $x(U) \subset \mathbb{Q}_{p}^{d}$. 
Theorem 1.4.1. Let $\omega \neq 0$ be an invariant $d$-form on $G_{\mathbb{Q}_{p}}$. Let $[\omega]$ be the corresponding class in $H^{d}\left(\mathfrak{g}, \mathbb{Q}_{p}\right)$. Then

$$
\mu_{[\omega]}^{\mathrm{coh}}=\mu_{\omega}^{\mathrm{Tam}}
$$

as measures on $G\left(\mathbb{Z}_{p}\right)$.

Proof. It suffices to check that

$$
\mu_{[\omega]}^{\mathrm{coh}}(\mathcal{G})=\mu_{\omega}^{\operatorname{Tam}}(\mathcal{G})
$$

for some open compact subgroup $\mathcal{G} \subset G\left(\mathbb{Q}_{p}\right)$. As $\omega$ is unique up to scaling, it also suffices to check the assertion for some $\omega$.

Let

$$
G\left(p \mathbb{Z}_{p}\right):=\operatorname{ker}\left(G\left(\mathbb{Z}_{p}\right) \rightarrow G\left(\mathbb{F}_{p}\right)\right) .
$$

This is a standard group in the sense of Serre [S] (see also the detailed discussion in [HKN, Section 2.2]). Let $t_{1}, \ldots, t_{d}$ be coordinates of the formal group $\hat{G}$, the completion of $G$ along $e$. Then

$$
t=\left(t_{1}, \ldots, t_{d}\right): G\left(p \mathbb{Z}_{p}\right) \rightarrow \mathbb{Z}_{p}^{d}
$$

is well defined with image $\left(p \mathbb{Z}_{p}\right)^{d}$. The valuation

$$
\omega(x)=\sup _{i}\left|t_{i}(x)\right|_{p}
$$

turns $G\left(p \mathbb{Z}_{p}\right)$ into a $p$-valued group. Let $\mathcal{G}$ be its saturated subgroup, described as follows. In order to unify notation, let $q=p$ for $p \neq 2$, and let $q=4$ for $p=2$. By [HKN, Lemma 2.2.2], we have

$$
t(\mathcal{G})=\left(q \mathbb{Z}_{p}\right)^{d} .
$$

Recall that $\mathfrak{g}$ is the $\mathbb{Z}_{p}$-Lie algebra of the group scheme $G$. It is a free $\mathbb{Z}_{p}$-module of rank $d$. The exponential map induces a homeomorphism

$$
q \mathfrak{g} \rightarrow \mathcal{G} .
$$

By [HKN, Example 2.6.8], the $\mathbb{Z}_{p}$-Lie algebra $q \mathfrak{g}$ is nothing but the integral Lazard Lie algebra $\mathcal{L}(\mathcal{G})$ of $\mathcal{G}$. We have

$$
H^{d}\left(q \mathfrak{g}, \mathbb{Q}_{p}\right)=\bigwedge^{d}(q \mathfrak{g})^{\vee} \cong \mathbb{Z}_{p}
$$


Let $[\eta]$ be a generator. The main result of $[\mathrm{HKN}]$ is the compatibility of the Lazard isomorphism with integral structures. By [HKN, Theorem 3.1.1 and Example 3.3.1], we know that

$$
\operatorname{Laz}: H^{d}\left(\mathcal{G}, \mathbb{Z}_{p}\right) \rightarrow H^{d}\left(q \mathfrak{g}, \mathbb{Z}_{p}\right)
$$

is an isomorphism. In particular, $\operatorname{Laz}^{-1}([\eta])$ is a generator of $H^{d}\left(\mathcal{G}, \mathbb{Z}_{p}\right)$. This implies that

$$
\mu_{[\eta]}^{\mathrm{coh}}(\mathcal{G})=1
$$

We now turn to the Tamagawa measure. Let $\omega$ be a generator of the space of invariant algebraic $d$-forms on $G$. This space is a $\mathbb{Z}_{p}$-module of rank 1 ; hence, $\omega$ is well defined up to a factor in $\mathbb{Z}_{p}^{*}$. Restricting to the cotangent space at $e$, we get a generator of $\bigwedge^{d} \mathfrak{g}^{\vee}$. Hence, $q^{-d} \omega$ generates $\bigwedge^{d}(q \mathfrak{g})^{\vee}$. This implies that

$$
[\eta]=u q^{-d}[\omega] \in H^{d}\left(q \mathfrak{g}, \mathbb{Q}_{p}\right)
$$

with $u \in \mathbb{Z}_{p}^{*}$. Without loss of generality, $u=1$.

We now write $\omega$ in coordinates of the formal group. It has the form

$$
\omega=f d t_{1} \wedge \cdots d t_{n}
$$

with $f \in \mathbb{Z}_{p}\left[\left[t_{1}, \ldots, t_{n}\right]\right]$ such that $f(0)$ is a $p$-adic unit. Recall that $t(\mathcal{G})=$ $\left(q \mathbb{Z}_{p}\right)^{d}$. Hence,

$$
|f|_{p}=|f(0)|_{p}=1
$$

on $t(\mathcal{G})$, and the Tamagawa measure of $\mathcal{G}$ with respect to $\eta=q^{-d} \omega$ is

$$
\int_{\left(q \mathbb{Z}_{p}\right)^{d}}\left|q^{-d} f\right|_{p}=\int_{\left(q \mathbb{Z}_{p}\right)^{d}} q^{d}=1 .
$$

This finishes the proof.

REMARK 1.4.2. The above proof used the existence of a smooth model $G / \mathbb{Z}_{p}$. By [BLR, Section 7.1, Theorem 5] this is not a restriction.

Corollary 1.4.3. If $[\omega]$ is a $\mathbb{Z}_{p}$-generator of $H^{d}\left(\mathfrak{g}, \mathbb{Z}_{p}\right)$, then

$$
\mu_{[\omega]}^{\mathrm{coh}}\left(G\left(\mathbb{Z}_{p}\right)\right)=p^{-d}\left|G\left(\mathbb{F}_{p}\right)\right| .
$$

Proof. This is true for the local Tamagawa number $\tau_{\omega}$ (see [W, Theorem 2.2.5]). It also follows directly from the computation in the proof of Theorem 1.4.1. 
Proof of Theorem 1. Let $[\omega]$ be a generator of $H^{d}\left(\mathrm{Sl}_{n}, \mathbb{Z}\right)$; then, by Corollary 1.4.3,

$$
\mu_{[\omega]}^{\operatorname{coh}}\left(\operatorname{Sl}_{n}\left(\mathbb{Z}_{p}\right)\right)=p^{-d}\left|\operatorname{Sl}\left(\mathbb{F}_{p}\right)\right|=\left(1-p^{-2}\right)\left(1-p^{-3}\right) \cdots\left(1-p^{-n}\right)
$$

because

$$
\left|\mathrm{Sl}_{n}\left(\mathbb{F}_{p}\right)\right|=\left(p^{2}-1\right)\left(p^{3}-1\right) \cdots\left(p^{n}-1\right) p^{n(n-1) / 2}
$$

and $d=n^{2}-1$. Taking the product over all $p$ yields the formula of Theorem 1 .

\subsection{The global formula}

Let $G / \mathbb{Z}$ be as fixed in Section 1.1. Recall the class number $h_{G, K_{f}}$ from Definition 1.1.3. Let $S$ be a finite set of finite places including all primes of bad reduction of our data.

We want to define a cohomological Tamagawa number as a product of the local Tamagawa numbers for all places. However, the product does not converge in general. We have to introduce convergence factors.

Let $M$ be the motive of (the quasi-split inner form of) $G$ in the sense of [G, Chapter 1]. It has good reduction at $p \notin S$.

REMARK 1.5.1. The motive of the variety $G_{\mathbb{Q}}$ (e.g., in Voevodsky's triangulated category of motives) is a direct sum of Artin-Tate motives. It has the structure of a Hopf object. In can be shown that $M$ in the sense of Gross is the primitive part of $M(G)$ and independent of the choice of inner form. Details will be discussed elsewhere.

Definition 1.5.2. For $p \notin S$, let

$$
E_{p}(G, s)=\operatorname{det}\left(1-\operatorname{Fr}_{p}^{-1} p^{-s} \mid M\right)
$$

be the Euler factor of $M$ at $p$. The partial $L$-function of $G$ is defined as the Euler product

$$
L_{S}(G, s)=\prod_{p \notin S} E_{p}(G, s)^{-1} .
$$

This is $L_{S}(M, s)$ in the notation of [G, Section 9]. Under Assumption 1.1.6, the function has an analytic continuation (with no pole) to $s=1$ (see [G, Proposition 9.4]).

LEMMA 1.5.3. For almost all $p$,

$$
E_{p}(G, 1)=p^{-d}\left|G\left(\mathbb{F}_{p}\right)\right|
$$


Proof. For almost all $p, G_{\mathbb{F}_{p}}$ is quasi-split reductive. In this case, use [G, Section 3].

EXAMPLE 1.5.4. Let $K$ be a number field with ring of integers $\mathcal{O}$. If $G=\operatorname{Res}_{\mathcal{O} / \mathbb{Z}} \mathbb{G}_{m}$ is as in Example 1.1.7, then $L_{S}(M, s)=\zeta_{K, S}(s)$ is the partial Dedekind $\zeta$-function. For $G=\operatorname{Res}_{\mathcal{O} / \mathbb{Z}} \mathbb{G}_{m} / \mathbb{G}_{m}$, it is $\zeta_{K, S}(s) / \zeta_{S}(s)$. In particular, it is regular at $s=1$. If $G=H$ is as in Example 1.1.8 (kernel of the reduced norm of a central simple algebra over a number field $F$ ), then it is an inner form of $\mathrm{SL}_{n, F}$, and hence

$$
L_{S}(G, s)=L_{S}\left(\mathrm{SL}_{n, F}, s\right)=\prod_{i=1}^{n-1} \zeta_{F, S}(s+i) .
$$

Again, it is regular at $s=1$.

Definition 1.5.5. Let $[\omega] \in H^{d}(\operatorname{Lie}(G), \mathbb{Q})$. Then the cohomological Tamagawa number is defined as

$$
\begin{aligned}
& \tau^{\mathrm{coh}}\left(G, K_{f}\right) \\
& \quad=L_{S}(G, 1)^{-1}\left(\prod_{p \notin S} E_{p}(G, 1)^{-1} \mu_{[\omega]}^{\mathrm{coh}}\left(G_{p}^{c}\right)\right)\left(\prod_{p \in S} \mu_{[\omega]}^{\mathrm{coh}}\left(G_{p}^{c}\right)\right) \mu_{[\omega]}^{\mathrm{coh}}\left(\coprod_{g \in H_{G}, K_{f}} \Gamma_{g} \backslash G(\mathbb{R})\right) .
\end{aligned}
$$

For almost all $p$,

$$
E_{p}(G, 1)^{-1} \mu_{[\omega]}^{\mathrm{coh}}\left(G_{p}^{c}\right)=1
$$

by Corollary 1.4.3 and Lemma 1.5.3. Hence, the product converges. As usual, $\tau^{\mathrm{coh}}\left(G, K_{f}\right)$ is independent of the choices of $S$ and $[\omega]$.

REMARK 1.5.6. If $G_{\mathbb{Q}}$ is semisimple, then the convergence factors are not necessary. We have

$$
\tau^{\mathrm{coh}}\left(G, K_{f}\right)=\mu_{[\omega]}^{\mathrm{coh}}\left(\coprod_{g \in H_{G, K_{f}}} \Gamma_{g} \backslash G(\mathbb{R})\right) \prod_{p<\infty} \mu_{[\omega]}^{\mathrm{coh}}\left(G_{p}^{c}\right) .
$$

If, in addition, $G^{i}(\mathbb{R})$ is noncompact for all simple factors of $G_{\mathbb{Q}}$, then by Example 1.1.5, we have

$$
\tau^{\operatorname{coh}}\left(G, K_{f}\right)=\mu_{[\omega]}^{\mathrm{coh}}(\Gamma \backslash G(\mathbb{R})) \prod_{p<\infty} \mu_{[\omega]}^{\mathrm{coh}}\left(G_{p}^{c}\right) .
$$

This includes the case of our Example 1.1.8. 
Let $\tau^{\operatorname{Tam}}(G)$ be the Tamagawa number of the group $G$ in the sense of Weil, that is, the volume of $G(\mathbb{Q}) \backslash G(\mathbb{A})$ with respect to the global Tamagawa measure $\mu^{\text {Tam }}$. Recall from Lemma 1.1.2 that

$$
G(\mathbb{Q}) \backslash G(\mathbb{A}) / K_{f}=\coprod_{g \in H_{G, K_{f}}} \Gamma_{g} \backslash G(\mathbb{R})
$$

THEOREM 1.5.7. One has the equality

$$
\tau^{\operatorname{Tam}}(G)=\tau^{\operatorname{coh}}\left(G, K_{f}\right) .
$$

Proof. Recall that $G_{\infty}^{c}=G(\mathbb{R})$. We have

$$
\tau^{\operatorname{Tam}}(G)=\mu^{\operatorname{Tam}}(G(\mathbb{A}) / G(\mathbb{Q}))=\sum_{g \in H_{G, K_{f}}} \mu^{\operatorname{Tam}}\left(\prod_{p<\infty} G_{p}^{c} \times \Gamma_{g} \backslash G(\mathbb{R})\right) .
$$

By definition of $\mu^{\text {Tam }}$, the assertion now follows from the local identities of Proposition 1.2.7 and Theorem 1.4.1.

REMARK 1.5.8. Rationally, the Lazard isomorphism exists for all choices of $G_{p}^{c}$, and hence the formulation of the theorem is independent of the choice of model $G$. By [BLR, Section 7.1, Theorem 5], all reductive groups $G_{\mathbb{Q}}$ allow a smooth model $G$ over $\mathbb{Z}$ in the sense that we need.

COROLlary 1.5.9. If $G_{\mathbb{Q}}$ is semisimple and simply connected, then

$$
1=\tau^{\operatorname{coh}}\left(G, K_{f}\right)
$$

Proof. In this case, the Tamagawa number $\tau^{\operatorname{Tam}}(G)$ equals 1 (see $[\mathrm{PR}$, Section 5.3] for a discussion of results on Tamagawa numbers).

This includes the case of our Example 1.1.8.

\section{$\S 2$. Comparison with the Bloch-Kato conjecture in the case of tori}

\subsection{Notation}

Let $T$ be an algebraic torus of dimension $d$ over $\mathbb{Q}$. For each $\mathbb{Q}$-algebra $A$, we let $T(A)$ be the group of $A$-rational points. We denote by

$$
X^{*}:=\operatorname{Hom}_{\overline{\mathbb{Q}}}\left(T, \mathbb{G}_{m}\right), \quad X_{*}:=\operatorname{Hom}_{\overline{\mathbb{Q}}}\left(\mathbb{G}_{m}, T\right)
$$


the group of characters (resp., cocharacters) of $T$ defined over $\overline{\mathbb{Q}}$. For each field $\mathbb{Q} \subset K$, we denote by

$$
X_{K}^{*}:=\operatorname{Hom}_{K}\left(T \times_{\mathbb{Q}} K, \mathbb{G}_{m}\right), \quad X_{* K}:=\operatorname{Hom}_{K}\left(\mathbb{G}_{m}, T \times_{\mathbb{Q}} K\right)
$$

the group of characters (resp., cocharacters) defined over $K$. Let

$$
r:=\operatorname{rk} X_{\mathbb{Q}}^{*}, \quad r_{p}:=\operatorname{rk} X_{\mathbb{Q}_{p}}^{*}, \quad r_{\infty}=\operatorname{rk} X_{\mathbb{R}}^{*}
$$

be the $\mathbb{Q}$-rank, $\mathbb{Q}_{p}$-rank, and $\mathbb{R}$-rank of $T$, respectively.

Assumption 2.1.1. To avoid problems with the pole of the Riemann zeta function at 1 , we will consider only tori of $\mathbb{Q}$-rank 0 .

We denote the motive of $T$ by $V:=h_{1}(T)$. Considered as an Artin-Tate motive, this is $V=X_{*} \otimes \mathbb{Q}(1)$.

REMARK 2.1.2. As in Section 1.5, this is the motive of $T$ in the sense of Gross, that is, the dual of the primitive part of $M(T)$ in a suitable category of Artin-Tate motives.

We can recover the $N$-torsion $T[N](\overline{\mathbb{Q}})$ from $X_{*}(1)$ by observing that

$$
T[N](\overline{\mathbb{Q}})=\operatorname{Hom}_{\overline{\mathbb{Q}}}\left(X^{*} \otimes \mathbb{Z} / N \mathbb{Z}, \mathbb{G}_{m}\right) \cong X_{*} \otimes \mu_{N}
$$

In particular, we can identify the Tate module of $T$ with

$$
X_{*} \otimes \mathbb{Z}_{p}(1) \cong \lim _{n} T\left[p^{n}\right](\overline{\mathbb{Q}}) .
$$

The following sets of points of $T$ play an important role in the identification of the motivic points of $V$. By Assumption 2.1.1, we have

$$
T^{1}(\mathbb{A}):=\bigcap_{\chi \in X_{\mathbb{Q}}^{*}} \operatorname{ker}\left(\|\chi\|_{\mathbb{A}}\right)=T(\mathbb{A}),
$$

which implies that $T(\mathbb{A}) / T(\mathbb{Q})$ is compact. For each finite place $v$ of $\mathbb{Q}$, we define the maximal compact subtorus $T_{v}^{c} \subset T\left(\mathbb{Q}_{v}\right)$ by

$$
T_{v}^{c}:=\bigcap_{\chi \in X_{\mathbb{Q} v}^{*}} \operatorname{ker}\left(|\chi|_{v}\right),
$$

where $|\cdot|_{v}$ is the norm on $\mathbb{Q}_{v}$ normalized by $|p|_{p}=1 / p$. We have

$$
0 \rightarrow T_{v}^{c} \rightarrow T\left(\mathbb{Q}_{v}\right) \rightarrow \mathbb{Z}^{r_{v}} \rightarrow 0
$$


Define

$$
\Gamma:=T(\mathbb{Q}) \cap\left(T(\mathbb{R}) \times \prod_{p} T_{p}^{c}\right) \subset T(\mathbb{A}) .
$$

(This agrees with the notation of Section 1.1; we have $\Gamma=\Gamma_{g}$ for all $g$ because $T$ is commutative.) By Dirichlet's unit theorem, $\Gamma$ is of the form

$$
\Gamma=\left(T(\mathbb{Q}) \cap \prod_{p} T_{p}^{c}\right) \times E,
$$

where $E$ is a free group of rank $r_{\infty}$ (recall that $r=0$ by Assumption 2.1.1) and $\left(T(\mathbb{Q}) \cap \prod_{p} T_{p}^{c}\right)$ is a finite group.

Definition 2.1.3. Let $M$ be an abelian group; then we denote the $p$-adic completion by

$$
M^{\wedge p}:=\longleftarrow_{n}^{\lim } M / p^{n} M
$$

and the profinite completion by

$$
M^{\wedge}:=\lim _{N} M / N M
$$

Consider the Kummer sequence for $T$,

$$
0 \rightarrow T\left[p^{n}\right] \rightarrow T \stackrel{\left[p^{n}\right]}{\longrightarrow} T \rightarrow 0 .
$$

LEMma 2.1.4. For each field $k \supset \mathbb{Q}$, the Kummer sequence induces isomorphisms

$$
T(k)^{\wedge p} \cong H^{1}\left(k, X_{*} \otimes \mathbb{Z}_{p}(1)\right)
$$

and

$$
T(k)^{\wedge} \cong H^{1}\left(k, X_{*} \otimes \widehat{\mathbb{Z}}(1)\right) .
$$

Proof. The Kummer sequence induces

$$
0 \rightarrow T(k) / N T(k) \rightarrow H^{1}(k, T[N]) \rightarrow H^{1}(k, T)[N] \rightarrow 0,
$$

and taking the inverse limit, we have

$$
\lim _{n} H^{1}(k, T)\left[p^{n}\right]=0 \quad\left(\text { resp. }, \underset{\lim _{N}}{H^{1}}(k, T)[N]=0\right)
$$

as the transition maps are multiplication by $p$ and $H^{1}(k, T)$ is finite. As $\underset{\longleftarrow}{\lim }$ is left-exact, this implies the isomorphism. 


\section{2. $\mathbb{R}$-valued points of tori}

We consider the motive $h_{1}(T)$. The Betti realization of $h_{1}(T)$ is given by $h_{1}(T)_{B}=X_{*} \otimes \mathbb{Q}(1)$, which contains $X_{*}(1)$ as a lattice. The de Rham realization is $h_{1}(T)_{\mathrm{dR}}=\operatorname{Lie} T$, which has $\mathrm{Fil}^{0} h_{1}(T)_{\mathrm{dR}}=0$. Bloch and Kato [BK, (5.6)] consider $D_{\infty}:=h_{1}(T)_{\mathrm{dR}, \mathbb{R}}=\operatorname{Lie} T_{\mathbb{R}}$ and define the $\mathbb{R}$-valued points of the motive $h_{1}(T)$ by

$$
A(\mathbb{R})=\left(D_{\infty} \otimes_{\mathbb{R}} \mathbb{C} /\left(\mathrm{Fil}^{0} D_{\infty} \otimes_{\mathbb{R}} \mathbb{C}+X_{*}(1)\right)\right)^{+} .
$$

Proposition 2.2.1. The $\mathbb{R}$-valued points of $h_{1}(T)$ are given by

$$
A(\mathbb{R})=T(\mathbb{R}),
$$

and via the identification $D_{\infty} \cong \operatorname{Lie} T(\mathbb{R})$, the natural map

$$
D_{\infty} \rightarrow A(\mathbb{R})
$$

is the exponential map.

Proof. In our case, $D_{\infty} / \operatorname{Fil}^{0} D_{\infty} \cong \operatorname{Lie} T(\mathbb{R})$, so that $D_{\infty} \otimes_{\mathbb{R}} \mathbb{C} \cong \operatorname{Lie} T(\mathbb{C})$. We have an exact sequence

$$
0 \rightarrow X_{*}(1) \rightarrow X_{*} \otimes \mathbb{C} \rightarrow T(\mathbb{C}) \rightarrow 0,
$$

where the last map is the exponential. Hence, $X_{*} \otimes \mathbb{C} \cong \operatorname{Lie} T(\mathbb{C})$, and we get

$$
A(\mathbb{R})=\left(\operatorname{Lie} T(\mathbb{C}) / X_{*}(1)\right)^{+}=(T(\mathbb{C}))^{+}=T(\mathbb{R}) .
$$

\section{3. $\mathbb{Q}_{p}$-valued points of tori}

The aim of this section is to identify the $\mathbb{Q}_{p}$-valued motivic points. The $\mathbb{Q}_{p}$-valued motivic points are by definition (see [BK, (5.6)])

$$
A\left(\mathbb{Q}_{p}\right):=H_{f}^{1}\left(\mathbb{Q}_{p}, X_{*} \otimes \widehat{\mathbb{Z}}(1)\right) .
$$

Theorem 2.3.1. The $\mathbb{Q}_{p}$-valued motivic points of the motive $V=h_{1}(T)$ are given by

$$
A\left(\mathbb{Q}_{p}\right)=T_{p}^{c} .
$$

As a first step, we identify the torsion subgroup of $A\left(\mathbb{Q}_{p}\right)$.

Lemma 2.3.2. The torsion subgroup of $A\left(\mathbb{Q}_{p}\right)$ coincides with the one of $T_{p}^{c}$ :

$$
\left(T_{p}^{c}\right)_{\text {tors }}=T\left(\mathbb{Q}_{p}\right)_{\text {tors }}=A\left(\mathbb{Q}_{p}\right)_{\text {tors }}
$$


Proof. By definition of $H_{f}^{1}\left(\mathbb{Q}_{p}, X_{*} \otimes \widehat{\mathbb{Z}}(1)\right)$, the torsion coincides with the torsion in

$$
H^{1}\left(\mathbb{Q}_{p}, X_{*} \otimes \widehat{\mathbb{Z}}(1)\right)=\prod_{l} H^{1}\left(\mathbb{Q}_{p}, X_{*} \otimes \mathbb{Z}_{l}(1)\right) .
$$

The torsion in $H^{1}\left(\mathbb{Q}_{p}, X_{*} \otimes \mathbb{Z}_{l}(1)\right)$ is $H^{0}\left(\mathbb{Q}_{p}, X_{*}(1) \otimes \mathbb{Q}_{l} / \mathbb{Z}_{l}\right)$ because we have an exact sequence

$$
0 \rightarrow H^{0}\left(\mathbb{Q}_{p}, X_{*}(1) \otimes \mathbb{Q}_{l} / \mathbb{Z}_{l}\right) \rightarrow H^{1}\left(\mathbb{Q}_{p}, X_{*} \otimes \mathbb{Z}_{l}(1)\right) \rightarrow H^{1}\left(\mathbb{Q}_{p}, X_{*} \otimes \mathbb{Q}_{l}(1)\right),
$$

where the first zero appears as $H^{0}\left(\mathbb{Q}_{p}, X_{*} \otimes \mathbb{Q}_{l}(1)\right)=0$ for weight reasons. On the other hand,

$$
T\left[l^{\infty}\right](\overline{\mathbb{Q}})=\operatorname{Hom}_{\overline{\mathbb{Q}}}\left(X^{*}, \mu_{l \infty}\right)=X_{*}(1) \otimes \mathbb{Q}_{l} / \mathbb{Z}_{l} .
$$

This gives $H^{0}\left(\mathbb{Q}_{p}, X_{*}(1) \otimes \mathbb{Q}_{l} / \mathbb{Z}_{l}\right)=T\left[l^{\infty}\right]\left(\mathbb{Q}_{p}\right)$. To conclude, note that the exact sequence $(6)$ implies that $T_{p}^{c}\left[l^{\infty}\right]=T\left[l^{\infty}\right]\left(\mathbb{Q}_{p}\right)$.

LEMмa 2.3.3. One has, for $l \neq p$,

$$
H_{f}^{1}\left(\mathbb{Q}_{p}, V_{l}\right)=0 .
$$

In particular, $H_{f}^{1}\left(\mathbb{Q}_{p}, X_{*} \otimes \mathbb{Z}_{l}(1)\right)$ is torsion.

Proof. Let $I_{p}$ be the inertia group at $p$; then, by definition (see $[\mathrm{BK}$, $(3.7 .1)]), H_{f}^{1}\left(\mathbb{Q}_{p}, V_{l}\right)$ is given by the cokernel of the map

$$
V_{l}^{I_{p}} \stackrel{1-\mathrm{Fr}_{p}^{-1}}{\longrightarrow} V_{l}^{I_{p}}
$$

But $V=X_{*} \otimes \mathbb{Q}(1)$ is the Tate twist of an Artin motive, so that $\operatorname{Fr}_{p}^{-1}$ does not have 1 as an eigenvalue. This implies that $1-\mathrm{Fr}_{p}^{-1}$ is injective, hence surjective.

We have, by Lemma 2.1.4,

$$
T\left(\mathbb{Q}_{p}\right)^{\wedge p} \otimes \mathbb{Q}_{p} \cong H^{1}\left(\mathbb{Q}_{p}, V_{p}\right) .
$$

In order to identify $H_{f}^{1}\left(\mathbb{Q}_{p}, V_{p}\right)$ in $T\left(\mathbb{Q}_{p}\right)^{\wedge p} \otimes_{\mathbb{Z}_{p}} \mathbb{Q}_{p}$, we need the following.

Lemma 2.3.4. Denote by $v_{p}: \mathbb{Q}_{p}^{*} \rightarrow \mathbb{Z}$ the $p$-adic valuation; then one has an exact sequence

$$
0 \rightarrow T_{p}^{c \wedge p} \rightarrow T\left(\mathbb{Q}_{p}\right)^{\wedge p} \rightarrow \mathbb{Z}_{p}^{r_{p}} \rightarrow 0
$$


In particular,

$$
T_{p}^{c \wedge p} \otimes_{\mathbb{Z}_{p}} \mathbb{Q}_{p}=\bigcap_{\chi \in X_{\mathbb{Q}_{p}}^{*}} \operatorname{ker}\left(T\left(\mathbb{Q}_{p}\right)^{\wedge p} \otimes_{\mathbb{Z}_{p}} \mathbb{Q}_{p} \stackrel{v_{p} \circ \chi}{\longrightarrow} \mathbb{Q}_{p}\right) .
$$

Proof. Consider the exact sequence

$$
0 \rightarrow T_{p}^{c} \rightarrow T\left(\mathbb{Q}_{p}\right) \rightarrow \mathbb{Z}^{r_{p}} \rightarrow 0
$$

As $\mathbb{Z}^{r_{p}}$ is free, this sequence splits as a sequence of abelian groups; hence, taking the $p$-adic completion is exact. This gives the sequence (9). Tensoring this with $\mathbb{Q}_{p}$ over $\mathbb{Z}_{p}$, we get

$$
0 \rightarrow\left(T_{p}^{c}\right)^{\wedge p} \otimes_{\mathbb{Z}_{p}} \mathbb{Q}_{p} \rightarrow T\left(\mathbb{Q}_{p}\right)^{\wedge p} \otimes_{\mathbb{Z}_{p}} \mathbb{Q}_{p} \rightarrow \mathbb{Q}_{p}^{r_{p}} \rightarrow 0 .
$$

This implies the result.

The next lemma identifies $H_{f}^{1}\left(\mathbb{Q}_{p}, V_{p}\right) \subset T\left(\mathbb{Q}_{p}\right)^{\wedge p} \otimes_{\mathbb{Z}_{p}} \mathbb{Q}_{p}$.

Lemma 2.3.5. Denote by $v_{p}: \mathbb{Q}_{p}^{*} \rightarrow \mathbb{Z}$ the $p$-adic valuation; then

$$
H_{f}^{1}\left(\mathbb{Q}_{p}, V_{p}\right)=\bigcap_{\chi \in X_{\mathbb{Q}_{p}}^{*}} \operatorname{ker}\left(T\left(\mathbb{Q}_{p}\right)^{\wedge p} \otimes \mathbb{Q}_{p} \stackrel{v_{p} \circ \chi}{\longrightarrow} \mathbb{Q}_{p}\right) .
$$

In particular,

$$
\left(T_{p}^{c}\right)^{\wedge p} \otimes_{\mathbb{Z}_{p}} \mathbb{Q}_{p}=H_{f}^{1}\left(\mathbb{Q}_{p}, V_{p}\right)
$$

Proof. Every $\chi \in X_{\mathbb{Q}_{p}}^{*}$ defines a map

$$
H^{1}\left(\mathbb{Q}_{p}, V_{p}\right) \stackrel{\chi}{\rightarrow} H^{1}\left(\mathbb{Q}_{p}, \mathbb{Q}_{p}(1)\right) .
$$

By Lemma 2.1.4, for $T=\mathbb{G}_{m}$ one has $\left(\mathbb{Q}_{p}^{*}\right)^{\wedge p} \otimes_{\mathbb{Z}_{p}} \mathbb{Q}_{p} \cong H^{1}\left(\mathbb{Q}_{p}, \mathbb{Q}_{p}(1)\right)$. By [HK1, Lemma A.1 and Corollary A.2], one has

$$
\left(\mathbb{Z}_{p}^{*}\right)^{\wedge p} \otimes_{\mathbb{Z}_{p}} \mathbb{Q}_{p} \cong H_{f}^{1}\left(\mathbb{Q}_{p}, \mathbb{Q}_{p}(1)\right)
$$

and that $H_{f}^{1}\left(\mathbb{Q}_{p}, \mathbb{Q}_{p}(1)\right)$ is the kernel of the valuation map

$$
\left(\mathbb{Q}_{p}^{*}\right)^{\wedge p} \otimes \mathbb{Q}_{p} \stackrel{v_{p}}{\longrightarrow} \mathbb{Q}_{p}
$$

On the other hand, $\chi$ defines $H_{f}^{1}\left(\mathbb{Q}_{p}, V_{p}\right) \stackrel{\chi}{\rightarrow} H_{f}^{1}\left(\mathbb{Q}_{p}, \mathbb{Q}_{p}(1)\right)$. Putting this information together, we obtain

$$
H_{f}^{1}\left(\mathbb{Q}_{p}, V_{p}\right) \subset \bigcap_{\chi \in X_{\mathbb{Q}_{p}}^{*}} \operatorname{ker}\left(T\left(\mathbb{Q}_{p}\right)^{\wedge p} \otimes \mathbb{Q}_{p} \stackrel{v_{p} \circ \chi}{\longrightarrow} \mathbb{Q}_{p}\right) .
$$


By Lemma 2.3.4, we get

$$
H_{f}^{1}\left(\mathbb{Q}_{p}, V_{p}\right) \subset T_{p}^{c} \otimes \mathbb{Q}_{p}
$$

To show equality, we consider the dimension of both sides. Via the BlochKato exponential $D_{\mathrm{dR}}\left(V_{p}\right) \cong H_{f}^{1}\left(\mathbb{Q}_{p}, V_{p}\right)$, we see that the $\mathbb{Q}_{p}$-dimension of $H_{f}^{1}\left(\mathbb{Q}_{p}, V_{p}\right)$ is $\operatorname{dim}_{\mathbb{Q}_{p}} V_{p}$. On the other hand, the Euler characteristic formula gives

$$
\sum_{i=0}^{2} \operatorname{dim}_{\mathbb{Q}_{p}} H^{i}\left(\mathbb{Q}_{p}, V_{p}\right)=-\operatorname{dim}_{\mathbb{Q}_{p}} V_{p},
$$

which implies that

$$
\operatorname{dim}_{\mathbb{Q}_{p}} H^{1}\left(\mathbb{Q}_{p}, V_{p}\right)=\operatorname{dim}_{\mathbb{Q}_{p}} V_{p}+\operatorname{dim}_{\mathbb{Q}_{p}} H^{0}\left(\mathbb{Q}_{p}, V_{p}\right)=\operatorname{dim}_{\mathbb{Q}_{p}} V_{p}+r_{p}
$$

With the identification $T\left(\mathbb{Q}_{p}\right)^{\wedge p} \otimes_{\mathbb{Z}_{p}} \mathbb{Q}_{p} \cong H^{1}\left(\mathbb{Q}_{p}, V_{p}\right)$ and the exact sequence (6), we see that $\operatorname{dim}_{\mathbb{Q}_{p}}\left(T_{p}^{c} \otimes_{\mathbb{Z}_{p}} \mathbb{Q}_{p}\right)=\operatorname{dim}_{\mathbb{Q}_{p}} V_{p}$. This proves the desired result.

LEMmA 2.3.6. Under the identification $T\left(\mathbb{Q}_{p}\right)^{\wedge p} \cong H^{1}\left(\mathbb{Q}_{p}, X_{*} \otimes \mathbb{Z}_{p}(1)\right)$ by the Kummer sequence, the subgroup $H_{f}^{1}\left(\mathbb{Q}_{p}, X_{*} \otimes \mathbb{Z}_{p}(1)\right) \subset H^{1}\left(\mathbb{Q}_{p}, X_{*} \otimes\right.$ $\left.\mathbb{Z}_{p}(1)\right)$ coincides with $\left(T_{p}^{c}\right)^{\wedge p}$.

Proof. Consider the diagram

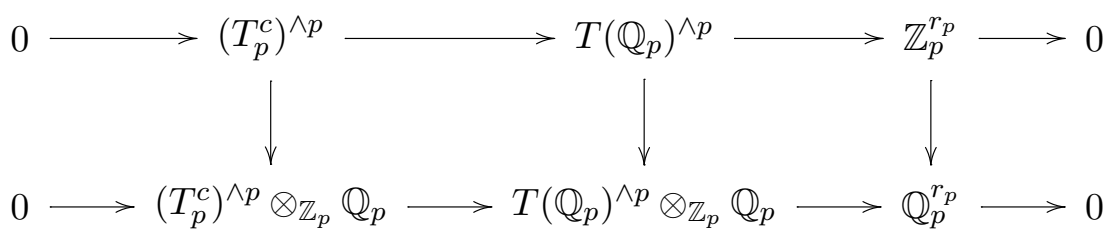

where both rows are exact by Lemma 2.3.4. Using the definition of $H_{f}^{1}\left(\mathbb{Q}_{p}\right.$, $\left.X_{*} \otimes \mathbb{Z}_{p}(1)\right)$ as the pullback of $H^{1}\left(\mathbb{Q}_{p}, X_{*} \otimes \mathbb{Z}_{p}(1)\right)$ to $H_{f}^{1}\left(\mathbb{Q}_{p}, V_{p}\right)$, we get that $\left(T_{p}^{c}\right)^{\wedge p} \cong H_{f}^{1}\left(\mathbb{Q}_{p}, X_{*} \otimes \mathbb{Z}_{p}(1)\right)$.

Finally, we can show Theorem 2.3.1.

Proof of Theorem 2.3.1. As both $T_{p}^{c}$ and $A\left(\mathbb{Q}_{p}\right)$ contain a subgroup of the form $\mathbb{Z}_{p}^{d}$ of finite index, it suffices to show that, for all $l$,

$$
\left(T_{p}^{c}\right)^{\wedge l} \cong A\left(\mathbb{Q}_{p}\right)^{\wedge l}
$$

For $l \neq p$ we have seen that both sides are torsion, and the claim follows from Lemma 2.3.2. For $l=p$ the claim follows from Lemma 2.3.6 and the definition of $A\left(\mathbb{Q}_{p}\right)$. 


\subsection{Comparison of the motivic} with the local Tamagawa measure

Let $\omega \neq 0$ be a $T$-invariant algebraic differential form defined over $\mathbb{Q}_{v}$ of top degree on $T$. This form defines the local Tamagawa measure $\mu_{\omega}^{\text {Tam }}$ on $T\left(\mathbb{Q}_{v}\right)$ (see Section 1.4) for all places $v$ with the property that

$$
\mu_{\omega}^{\operatorname{Tam}}\left(T_{p}^{c}\right)=p^{-\operatorname{dim} T} \# \mathcal{T}\left(\mathbb{F}_{p}\right)
$$

for almost all $p$. Here $\mathcal{T}$ is a smooth model of $T$ over $\mathbb{Z}_{p}$.

We next explain the motivic measures defined by Bloch and Kato on the local points of the motive $h_{1}(T)$. Choose once and for all a rational, topdegree translation-invariant differential form $\omega$ on $T$. This gives a linear form $\omega: \bigwedge^{d} \operatorname{Lie} T \rightarrow \mathbb{Q}$, and we denote by $\omega^{\vee} \in \bigwedge^{d} \operatorname{Lie} T$ the dual basis.

By definition [BK, Definition 5.9], the motivic measure $\mu_{\omega}^{\mathrm{BK}}$ on $A(\mathbb{R})=$ $T(\mathbb{R})$ equals $\mu_{\omega}^{\text {Tam }}$.

Next consider $A\left(\mathbb{Q}_{p}\right)=T_{p}^{c}$. Here the motivic measure $\mu_{\omega}^{\mathrm{BK}}$ is the Haar measure on $T_{p}^{c}$ normalized as follows. The Bloch-Kato exponential map induces an isomorphism

$$
\exp _{\mathrm{BK}}: \operatorname{Lie} T_{\mathbb{Q}_{p}} \cong H_{f}^{1}\left(\mathbb{Q}_{p}, X_{*} \otimes \mathbb{Q}_{p}(1)\right) \text {. }
$$

By definition of $A\left(\mathbb{Q}_{p}\right)=T_{p}^{c}$, a subgroup of finite index, say, $\mathcal{T}_{1} \subset A\left(\mathbb{Q}_{p}\right)$, is contained in $H_{f}^{1}\left(\mathbb{Q}_{p}, X_{*} \otimes \mathbb{Q}_{p}\right)$. Then

$$
\exp _{\mathrm{BK}}^{-1}\left(\mathcal{T}_{1}\right)=: \mathcal{T} \subset \operatorname{Lie} T_{\mathbb{Q}_{p}}
$$

is a $\mathbb{Z}_{p}$-lattice. Choose a basis $t_{1}, \ldots, t_{d}$ of $\mathcal{T}$; then we normalize $\mu_{p, \omega}^{\mathrm{BK}}$ by

$$
\mu_{p, \omega}^{\mathrm{BK}}\left(\mathcal{T}_{1}\right)=\left|\omega\left(t_{1} \wedge \cdots \wedge t_{d}\right)\right|_{p}
$$

We need the following information about $\exp _{\mathrm{BK}}$.

Lemma 2.4.1. The diagram

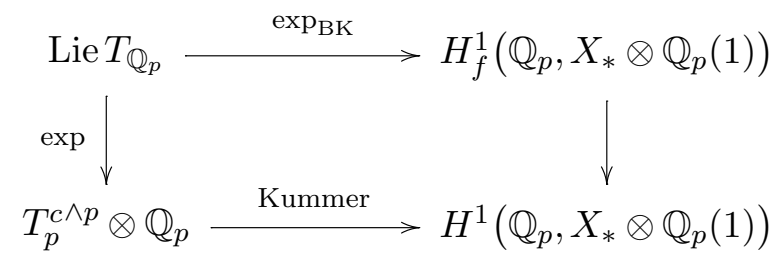

commutes. Here exp is the exponential map of $T_{p}^{c}$. 
Proof. After base change to the splitting field, one sees with [BK, Example 3.10.1] that $\exp _{\mathrm{BK}}$ is given by the exponential map.

Proposition 2.4.2. For all $v$, the motivic and the local Tamagawa measures coincide:

$$
\mu_{\omega}^{\mathrm{BK}}=\mu_{\omega}^{\mathrm{Tam}} .
$$

Proof. For $v=\infty$, both measures are defined in the same way, and there is nothing to show. For $v=p$, by Lemma 2.4.1, we have $\exp : \mathcal{T} \cong \mathcal{T}_{1}$. On $\mathcal{T}_{1}$ we have the translation-invariant form $\omega$, and the pullback $\exp ^{*} \omega$ is an invariant form on $\mathcal{T}$. We claim that this coincides with $\omega$. But exp induces the identity on the tangent spaces, so that $0^{*} \exp ^{*} \omega=e^{*} \omega$, where zero and $e$ are the unit sections of $\mathcal{T}$ and $\mathcal{T}_{1}$. As $e^{*} \omega$ is the linear form $\omega: \bigwedge^{d} \operatorname{Lie} T \rightarrow \mathbb{Q}$, the claim follows. Thus, the Tamagawa measure of $\mathcal{T}_{1}$ is $\omega\left(t_{1} \wedge \cdots \wedge t_{d}\right)$, where $t_{1}, \ldots, t_{d}$ is the basis of $\mathcal{T}$ chosen above.

\subsection{Global points of tori}

For any number field $K$, let $\operatorname{MAT}(K)$ be the $\mathbb{Q}$-linear abelian category of mixed Artin-Tate motives over $K$.

REMARK 2.5.1. Whereas the category of mixed motives in general is conjectural, the subcategory of Artin-Tate motives is well defined, for example, as (the opposed category of) the heart of the motivic $t$-structure on the full triangulated subcategory of Voevodsky's category $D M_{\mathrm{gm}}(\operatorname{Spec} K, \mathbb{Q})$ generated by Artin motives over $K$ and all pure Tate motives $\mathbb{Q}(n)$ for $n \in \mathbb{Z}$. It contains the homological motive of $T$. Its $h_{1}$ is given by $X_{*} \otimes \mathbb{Q}(1)$.

The realization functors attach to all objects of $\operatorname{MAT}(K)$, mixed Hodge structures, or $\operatorname{Gal}(\bar{K} / K)$-modules.

We put

$$
H_{\mathrm{mot}}^{1}(K, V)=\operatorname{Ext}_{\mathrm{MAT}(K)}^{1}\left(\mathbb{Q}(0), h_{1}(T)\right) .
$$

Recall [BK, p. 374] that the global points $A(\mathbb{Q})$ of the motive $V=h_{1}(T)$ are defined as follows. Let

$$
H_{\text {mot }, f}^{1}(\mathbb{Q}, V):=\operatorname{ker}\left(H_{\text {mot }}^{1}(\mathbb{Q}, V) \rightarrow \prod_{p<\infty} H^{1}\left(\mathbb{Q}_{p}, V_{p}\right) / H_{f}^{1}\left(\mathbb{Q}_{p}, V_{p}\right)\right)
$$

Then $A(\mathbb{Q}) \subset H_{f}^{1}\left(\mathbb{Q}, X_{*} \otimes \widehat{\mathbb{Z}}(1)\right)$ is the preimage of

$$
H_{\mathrm{mot}, f}^{1}(\mathbb{Q}, V) \subset H_{f}^{1}\left(\mathbb{Q}, X_{*} \otimes \widehat{\mathbb{Z}}(1)\right) \otimes_{\mathbb{Z}} \mathbb{Q}
$$

in $H_{f}^{1}\left(\mathbb{Q}, X_{*} \otimes \widehat{\mathbb{Z}}(1)\right)$. 
TheOREM 2.5.2. Recall that $\Gamma=T(\mathbb{Q}) \cap\left(T(\mathbb{R}) \times \prod_{p} T_{p}^{c}\right)$; then the global points of the motive $V=h_{1}(T)$ are

$$
A(\mathbb{Q})=\Gamma .
$$

For the proof we need several lemmas.

Lemma 2.5.3. One has

$$
H_{\mathrm{mot}}^{1}(\mathbb{Q}, V) \cong T(\mathbb{Q}) \otimes_{\mathbb{Z}} \mathbb{Q}
$$

and

$$
H_{\text {mot }, f}^{1}(\mathbb{Q}, V) \cong \Gamma \otimes_{\mathbb{Z}} \mathbb{Q} .
$$

Proof. Let $t \in T(\mathbb{Q})$. By the Abel-Jacobi map, the homologically trivial cycle $[t]-[1]$ induces an element of $\operatorname{Ext}_{\mathrm{MAT}}^{1}\left(\mathbb{Q}(0), h_{1}(T)\right)$. This defines a natural map, $T(\mathbb{Q}) \otimes_{\mathbb{Z}} \mathbb{Q} \rightarrow H_{\text {mot }}^{1}(\mathbb{Q}, V)$. Let $K / \mathbb{Q}$ be the splitting field of $T$; then $T(K)=\left(K^{*}\right)^{d}$, and hence

$$
H_{\mathrm{mot}}^{1}(K, V) \cong T(K) \otimes_{\mathbb{Z}} \mathbb{Q} .
$$

Using $H_{\text {mot }}^{1}(\mathbb{Q}, V)=H_{\text {mot }}^{1}(K, V)^{\operatorname{Gal}(K / \mathbb{Q})}$, the result for $H_{\text {mot }}^{1}(\mathbb{Q}, V)$ follows. With this result and Lemma 2.3.5, we have a Cartesian diagram

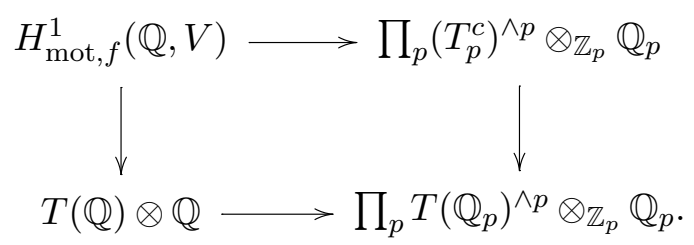

On the other hand, consider the exact sequence

$$
0 \rightarrow \Gamma \rightarrow T(\mathbb{Q}) \stackrel{\prod v_{p}}{\longrightarrow} \prod_{p} T\left(\mathbb{Q}_{p}\right)^{\wedge p} /\left(T_{p}^{c}\right)^{\wedge p}=\prod_{p} \mathbb{Z}_{p}^{r_{p}} .
$$

If we tensor with $\mathbb{Q}$ and use $\left(\prod_{p} \mathbb{Z}^{r_{p}}\right) \otimes \mathbb{Q} \subset \prod_{p} \mathbb{Q}_{p}^{r_{p}}$, we get

$$
0 \rightarrow \Gamma \otimes_{\mathbb{Z}} \mathbb{Q} \rightarrow T(\mathbb{Q}) \otimes_{\mathbb{Z}} \mathbb{Q} \stackrel{\prod v_{p} \otimes \mathrm{id}}{\longrightarrow} \prod_{p} \mathbb{Q}_{p}^{r_{p}}
$$

In particular, diagram (10) is Cartesian with $H_{\mathrm{mot}, f}^{1}(\mathbb{Q}, V)$ replaced by $\Gamma \otimes_{\mathbb{Z}} \mathbb{Q}$, which proves our claim. 
Lemma 2.5.4. There is a Cartesian diagram

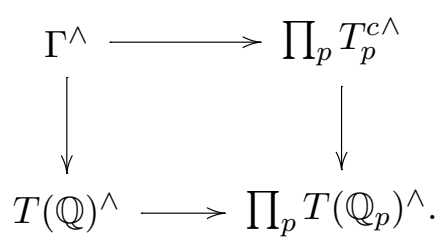

Proof. By definition, the diagram

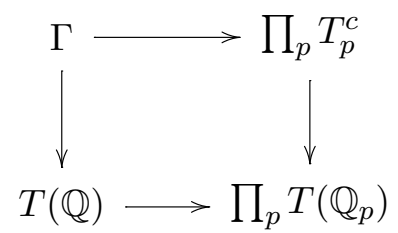

is Cartesian. Note that the cokernel of $\prod_{p} T_{p}^{c} \rightarrow \prod_{p} T\left(\mathbb{Q}_{p}\right)$ is $\prod_{p} \mathbb{Z}^{r_{p}}$ and hence torsion free. By $(11)$, the cokernel of $\Gamma \rightarrow T(\mathbb{Q})$ is also torsion free. Moreover, as the $N$-multiplication on a product is the product of the $N$ multiplications, the exact sequence

$$
\prod_{p} T(\mathbb{Q}) \stackrel{[N]}{\longrightarrow} \prod_{p} T(\mathbb{Q}) \rightarrow \prod_{p}(T(\mathbb{Q}) / N T(\mathbb{Q})) \rightarrow 0
$$

shows that $\left(\prod_{p} T(\mathbb{Q})\right) \otimes \mathbb{Z} / N \cong \prod_{p}(T(\mathbb{Q}) \otimes \mathbb{Z} / N)$. This implies that, for each $N \in \mathbb{Z}$, we have a Cartesian diagram

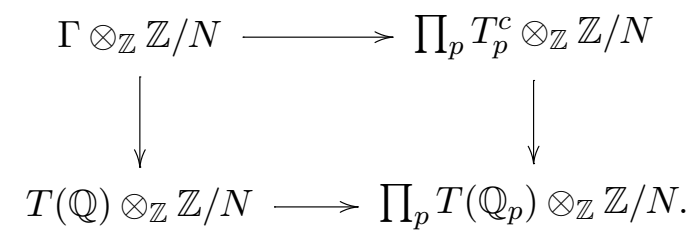

Passing to $\lim _{N}$ and observing that this commutes with products and finite fiber products, hence with Cartesian diagrams, gives the claim.

Corollary 2.5.5. One has

$$
H_{f}^{1}\left(\mathbb{Q}, X_{*} \otimes \widehat{\mathbb{Z}}(1)\right)=\Gamma^{\wedge}=\Gamma \otimes_{\mathbb{Z}} \widehat{\mathbb{Z}}
$$


Proof. The first equality follows from Lemma 2.5.4, the definition of $H_{f}^{1}\left(\mathbb{Q}, X_{*} \otimes \widehat{\mathbb{Z}}(1)\right)$ by the Cartesian diagram

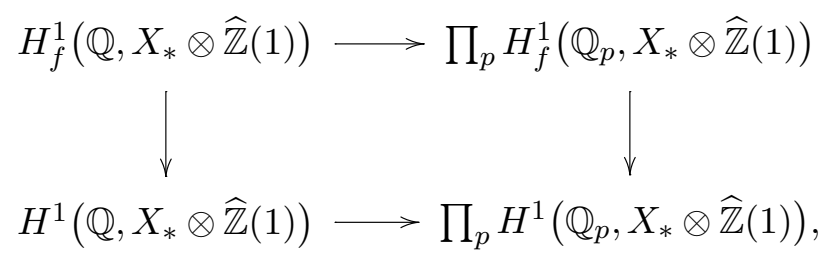

and the identifications in Theorem 2.3.1 and Lemma 2.1.4. The second equality follows from the fact that $\Gamma$ is a finitely generated abelian group, so that its profinite completion is given by $\Gamma \otimes_{\mathbb{Z}} \widehat{\mathbb{Z}}$.

Proof of Theorem 2.5.2. The global motivic points are by definition the fiber product of $H_{\text {mot }, f}^{1}(\mathbb{Q}, V)=\Gamma \otimes_{\mathbb{Z}} \mathbb{Q}\left(\right.$ Lemma 2.5.3) and $H_{f}^{1}\left(\mathbb{Q}, X_{*} \otimes\right.$ $\widehat{\mathbb{Z}}(1))=\Gamma \otimes_{\mathbb{Z}} \widehat{\mathbb{Z}}\left(\right.$ Corollary 2.5.5) over $H_{f}^{1}\left(\mathbb{Q}, X_{*} \otimes \widehat{\mathbb{Z}}(1)\right) \otimes \mathbb{Q}=\Gamma \otimes_{\mathbb{Z}} \mathbb{A}_{f}$. To finish the proof, we have to show that there is a Cartesian diagram

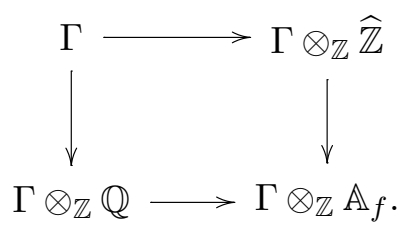

As $\Gamma$ is a finitely generated abelian group, we can prove this statement for the free part and the torsion part separately. For the free part, it follows from the standard Cartesian diagram

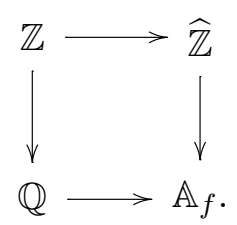

For the torsion part, it suffices to note that $\Gamma$ and $\Gamma \otimes_{\mathbb{Z}} \widehat{\mathbb{Z}}$ have obviously the same torsion.

\subsection{Global invariants}

Recall the definition of the classical Shafarevich group for tori (see [M, Theorem 9.11]):

$$
\amalg(T)=\operatorname{Ker}\left(H^{1}(\mathbb{Q}, T) \rightarrow \prod_{p \leq \infty} H^{1}\left(\mathbb{Q}_{p}, T\right)\right) .
$$


On the other hand, Bloch and Kato define a group $\amalg_{\mathrm{BK}}(M)$ for all motives $M / \mathbb{Q}$ (see [BK, (5.13)]). Using our identifications of local and global points, it reads

$$
\amalg_{\mathrm{BK}}\left(h_{1}(T)\right)=\operatorname{Ker}\left(\frac{H^{1}\left(\mathbb{Q}, X_{*}(1) \otimes \mathbb{Q} / \mathbb{Z}\right)}{\Gamma \otimes \mathbb{Q} / \mathbb{Z}} \rightarrow \bigoplus_{p \leq \infty} \frac{H^{1}\left(\mathbb{Q}_{p}, X_{*}(1) \otimes \mathbb{Q} / \mathbb{Z}\right)}{T_{p}^{c} \otimes \mathbb{Q} / \mathbb{Z}}\right),
$$

where by abuse of notation we put $T_{\infty}^{c}=T(\mathbb{R})$.

Definition 2.6.1. The class group of $T$ is

$$
\mathrm{Cl}(T):=\mathrm{Cl}(T, \Gamma):=\frac{T\left(\mathbb{A}_{f}\right)}{T(\mathbb{Q}) \prod_{p<\infty} T_{p}^{c}}
$$

We denote its order as $h_{\Gamma}$.

REMARK 2.6.2. $\mathrm{Cl}(T, \Gamma)$ is nothing but $H_{T, K_{f}}$ of Section 1.1. In particular, $h_{\Gamma}=h_{T, K_{f}}$ (see Definition 1.1.3), and the group is finite. If $T=$ $\operatorname{Res}_{K / \mathbb{Q}} \mathbb{G}_{m}$, then $\mathrm{Cl}(T)$ is the class group of $K$.

Our aim is to show the following.

Proposition 2.6.3. There is a natural short exact sequence

$$
0 \rightarrow \mathrm{Cl}(T) \rightarrow \amalg_{\mathrm{BK}}\left(h_{1}(T)\right) \rightarrow \amalg(T) \rightarrow 0
$$

Proof. Note that

$$
T\left(\mathbb{A}_{f}\right) / \prod_{p<\infty} T_{p}^{c} \cong \bigoplus_{p<\infty} T\left(\mathbb{Q}_{p}\right) / T_{p}^{c}
$$

(Take the direct limit over $S$-adeles for finite sets of places $S$.) By definition of $\mathrm{Cl}(T)$, this implies that

$$
0 \rightarrow T(\mathbb{Q}) / \Gamma \rightarrow \bigoplus_{p<\infty} T\left(\mathbb{Q}_{p}\right) / T_{p}^{c} \rightarrow \mathrm{Cl}(T) \rightarrow 0
$$

We abbreviate $I(T)$ for the middle group. Recall that $T\left(\mathbb{Q}_{p}\right) / T_{p}^{c} \cong \mathbb{Z}^{r_{p}}$ in our notation. Hence, $I(T)$ is torsion free. 
Let $n$ be a natural number. We get a commutative diagram with exact rows and columns:

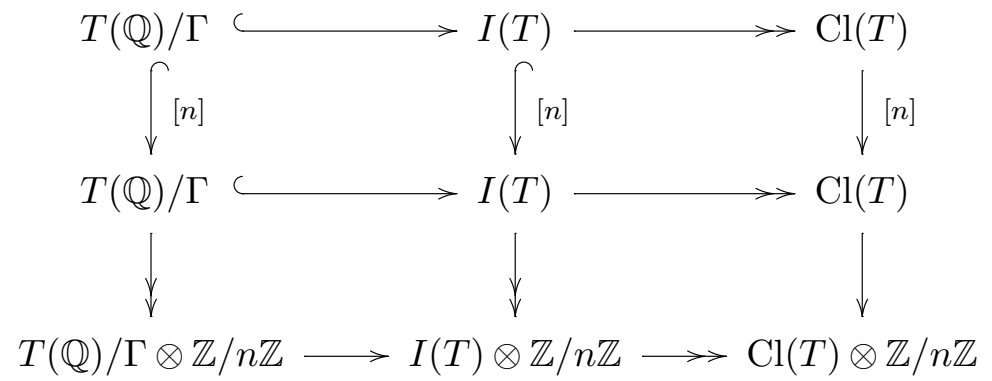

By the snake lemma, the kernel of the last line is isomorphic to $\mathrm{Cl}(T)[n]$. This implies that the sequence

$$
\begin{aligned}
0 \rightarrow \mathrm{Cl}(T)[n] & \rightarrow T(\mathbb{Q}) / \Gamma \otimes \mathbb{Z} / n \mathbb{Z} \rightarrow \bigoplus_{p} T\left(\mathbb{Q}_{p}\right) / T_{p}^{c} \otimes \mathbb{Z} / n \\
& \rightarrow \mathrm{Cl}(T) / n \mathrm{Cl}(T) \rightarrow 0
\end{aligned}
$$

is exact. We pass to the direct limit over $n$. Note that the transition map $\mathrm{Cl}(T)[n] \rightarrow \mathrm{Cl}(T)[n m]$ is the natural inclusion. As $\mathrm{Cl}(T)$ is finite, this means that

$$
\lim _{\longrightarrow} \mathrm{Cl}(T)[n]=\mathrm{Cl}(T) .
$$

On the other hand, the transition map $\mathrm{Cl}(T) / n \mathrm{Cl}(T) \rightarrow \mathrm{Cl}(T) / n m \mathrm{Cl}(T)$ is multiplication by $m$. Again, by finiteness, this means that

$$
\stackrel{\lim }{\longrightarrow} \mathrm{Cl}(T) / n \mathrm{Cl}(T)=0 .
$$

We have established the short exact sequence

$$
0 \rightarrow \mathrm{Cl}(T) \rightarrow \frac{T(\mathbb{Q}) \otimes \mathbb{Q} / \mathbb{Z}}{\Gamma \otimes \mathbb{Q} / \mathbb{Z}} \rightarrow \bigoplus_{p} \frac{T\left(\mathbb{Q}_{p}\right) \otimes \mathbb{Q} / \mathbb{Z}}{T_{p}^{c} \otimes \mathbb{Q} / \mathbb{Z}} \rightarrow 0
$$

Recall that by abuse of notation $T_{\infty}^{c}=T(\mathbb{R})$; hence, the equation remains valid when the sum runs through $p \leq \infty$.

By Kummer theory for the torus $T$, we have

$$
H^{1}(k, T(\bar{k})) \cong \frac{H^{1}\left(k, T(\bar{k})_{\text {tors }}\right)}{T(k) \otimes \mathbb{Q} / \mathbb{Z}}
$$


for any field $k \supset \mathbb{Q}$. Hence, the defining sequence for $\amalg(T)$ can be rewritten as

$$
0 \rightarrow \amalg(T) \rightarrow \frac{H^{1}\left(\mathbb{Q}, T(\overline{\mathbb{Q}})_{\text {tors }}\right)}{T(\mathbb{Q}) \otimes \mathbb{Q} / \mathbb{Z}} \rightarrow \prod_{p \leq \infty} \frac{H^{1}\left(\mathbb{Q}_{p}, T\left(\overline{\mathbb{Q}}_{p}\right)_{\text {tors }}\right)}{T\left(\mathbb{Q}_{p}\right) \otimes \mathbb{Q} / \mathbb{Z}}
$$

In this sequence, we can replace the product by a direct sum because all global cohomology classes are unramified almost everywhere, and unramified local classes vanish for tori.

Comparing this to the defining sequence of $\amalg_{\mathrm{BK}}$ yields a commutative diagram of exact sequences:

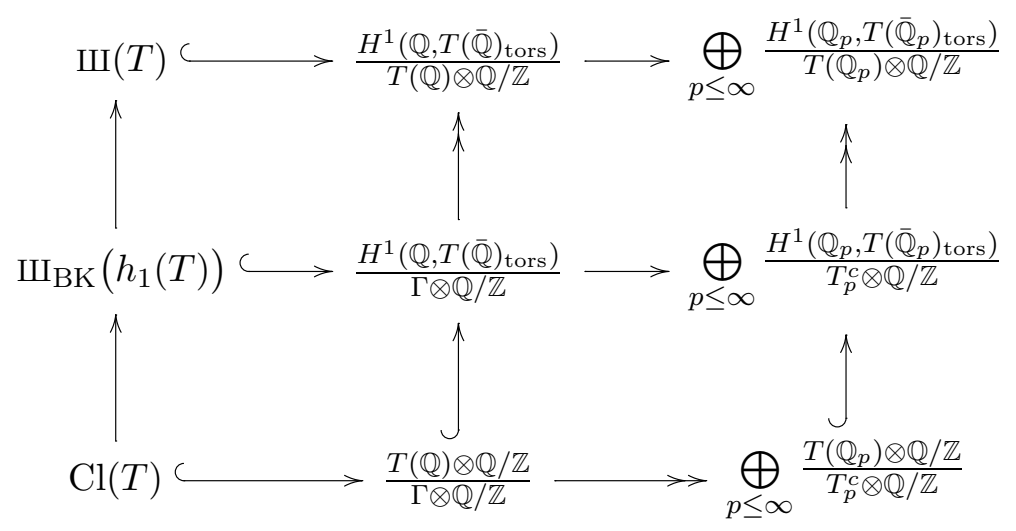

where the last line was shown in (12). The snake lemma gives the proposition.

Corollary 2.6.4. Let $i(T)$ be Ono's constant (see [O, Section 3.4]). Then

$$
\# \amalg_{\mathrm{BK}}\left(h_{1}(T)\right)=h_{\Gamma} \cdot i(T) .
$$

Proof. By definition, $i(T)$ is the order of

$$
\amalg^{\prime}(T)=\operatorname{Ker}\left(H^{1}(K / \mathbb{Q}, T(K))\right) \rightarrow H^{1}\left(K / \mathbb{Q}, T\left(\mathbb{A}_{K}\right)\right)
$$

for big enough $K$ (independent of this choice). By abuse of notation, let $T$ be a model of the torus $T / \mathbb{Q}$ over some open part of Spec $\mathbb{Z}$. By definition,

$$
H^{1}\left(K / \mathbb{Q}, T\left(\mathbb{A}_{K}\right)\right)=\underset{S}{\lim } \prod_{v \in S} H^{1}\left(K / \mathbb{Q}, T\left(K_{v}\right)\right) \times \prod_{v \notin S} H^{1}\left(K / \mathbb{Q}, T\left(\mathcal{O}_{v}\right)\right),
$$


where the limit is over finite sets of finite places of $K$ and $\mathcal{O}_{v}$ is the ring of integers of $K_{v}$. For any rational prime $p$, we choose a place $v$ of $K$ over $p$. Then

$$
\bigoplus_{v^{\prime} \mid p} H^{1}\left(K / \mathbb{Q}, T\left(K_{v^{\prime}}\right)\right) \cong H^{1}\left(K_{v} / \mathbb{Q}_{p}, T\left(K_{v}\right)\right) \text {. }
$$

As in the proof of Proposition 2.6.3, the classes in the image of $H^{1}(K / \mathbb{Q}$, $T(K))$ are unramified almost everywhere, and unramified local classes vanish for tori. Hence, we can replace the products by direct sums. Taking the direct limit over $S$, this means that

$$
0 \rightarrow \amalg^{\prime}(T) \rightarrow H^{1}(K / \mathbb{Q}, T(\mathbb{Q})) \rightarrow \bigoplus_{p} H^{1}\left(K_{v} / \mathbb{Q}, T\left(K_{v}\right)\right)
$$

Passing to the limit over $K$, we get the defining sequence for $\amalg(T)$.

We also need another global cohomological invariant.

Lemma 2.6.5. Let $T_{\mathbb{Q}}$ be a torus satisfying Assumption 1.1.6, that is, with $\mathbb{Q}$-rank zero. Then

$$
H^{1}\left(\mathbb{Q}, X^{*}\right) \cong H^{0}\left(\mathbb{Q}, X^{*} \otimes \mathbb{Q} / \mathbb{Z}\right)
$$

Proof. Under our assumption, this follows from the long exact sequence for the short exact sequence of discrete Galois modules,

$$
0 \rightarrow X^{*} \rightarrow X^{*} \otimes \mathbb{Q} \rightarrow X^{*} \otimes \mathbb{Q} / \mathbb{Z} \rightarrow 0
$$

\subsection{The Tamagawa number conjecture for tori}

Recall that $T / \mathbb{Q}$ is a torus with $\mathbb{Q}$-rank equal to zero. We now turn to stating the Bloch-Kato conjecture for the motive $h_{1}(T)=X_{*} \otimes \mathbb{Q}(1)$. Let $\omega$ be an invariant $d$-form on $T$. Recall the local measures $\mu_{\omega}^{\mathrm{BK}}$ on the local points $A\left(\mathbb{Q}_{p}\right)$ of the motive for $p \leq \infty$ (see Section 2.4). They define a product measure $\mu^{\mathrm{BK}}$ on

$$
\left(\prod_{p \leq \infty} A\left(\mathbb{Q}_{p}\right)\right) / A(\mathbb{Q})
$$

This global measure is independent of the choice of $\omega$.

REMARK 2.7.1. Note that $h_{1}(T)$ is pure of weight -1 . Hence, we have to use the refined definition (see [BK, Formula 5.9.1]) for the Tamagawa number. In the same way as in Definition 1.5.5 or in the classical Tamagawa measure, we introduce convergence factors. 
The Tamagawa number of $h_{1}(T)$ is

$$
\tau^{\mathrm{BK}}\left(h_{1}(T)\right)=\mu^{\mathrm{BK}}\left(\left(\prod_{p \leq \infty} A\left(\mathbb{Q}_{p}\right)\right) / A(\mathbb{Q})\right) .
$$

Theorem 2.7.2. Let $T$ be a torus of $\mathbb{Q}$-rank equal to zero. Then the Tamagawa number conjecture of Bloch and Kato [BK, Conjecture 5.15] holds for the motive $h_{1}(T)=X_{*} \otimes \mathbb{Q}(1)$; that is,

$$
\tau^{\mathrm{BK}}\left(h_{1}(T)\right)=\frac{\left.\# H^{0}\left(\mathbb{Q}, X^{*} \otimes \mathbb{Q} / \mathbb{Z}\right)\right)}{\# \amalg_{\mathrm{BK}}\left(h_{1}(T)\right)} .
$$

Proof of Theorem 2.7.2. We have already identified local points and local measures (see Proposition 2.4.2). Hence, $\mu^{\mathrm{BK}}=\mu^{\text {Tam }}$. Using in addition Corollary 2.6.4 and Lemma 2.6.5, the claim is equivalent to

$$
\mu^{\operatorname{Tam}}\left(T(\mathbb{R}) \prod_{p<\infty} T_{p}^{c} / \Gamma\right)=\frac{\# H^{1}\left(\mathbb{Q}, X^{*}\right)}{h_{\Gamma} i(T)} .
$$

On the other hand, the classical Tamagawa number formula for tori as proved by Ono [O, Section 5, Main Theorem] reads

$$
\mu^{\operatorname{Tam}}(T(\mathbb{A}) / T(\mathbb{Q}))=\frac{\# H^{1}\left(\mathbb{Q}, X^{*}\right)}{i(T)} .
$$

Together with the definition of $h_{\Gamma}$, this proves the theorem.

REMARK 2.7.3. This clears up a point we had been wondering about: where is the class number in the Tamagawa number conjecture?

Acknowledgment. We thank Burt Totaro for a remark on the existence of smooth models of reductive groups.

\section{REFERENCES}

[B] S. Bloch, A note on height pairings, Tamagawa numbers, and the BirchSwinnerton-Dyer conjecture, Invent. Math. 58 (1980), 65-76.

[BK] S. Bloch and K. Kato, " $L$-functions and Tamagawa numbers of motives" in The Grothendieck Festschrift, Vol. I, Progr. Math. 86, Birkhäuser, Boston, 1990, 333400 .

[Bo] A. Borel, Cohomologie de $\mathrm{SL}_{n}$ et valeurs de fonctions zeta aux points entiers, Ann. Sc. Norm. Supér. Pisa Cl. Sci. (5) 4 (1977), 613-636.

[BLR] S. Bosch, W. Lütkebohmert, and M. Raynaud, Néron Models, Ergeb. Math. Grenzgeb. (3) 21, Springer, Berlin, 1990. 
[CR] C. W. Curtis and I. Reiner, Methods of Representation Theory, Vol. II: With Applications to Finite Groups and Orders, Pure Appl. Math., Wiley, New York, 1987.

[F] J.-M. Fontaine, Valeurs spéciales des fonctions L de motifs, Astérisque 206 (1992), Séminaire Bourbaki, no. 751.

[G] B. H. Gross, On the motive of a reductive group, Invent. Math. 130 (1997), 287313.

[H] A. Huber, Poincaré duality for p-adic Lie groups, Arch. Math. 95 (2010), 509-517.

[HK1] A. Huber and G. Kings, Bloch-Kato conjecture and main conjecture of Iwasawa theory for Dirichlet characters, Duke Math. J. 119 (2003), 393-464.

[HK2] - A p-adic analogue of the Borel regulator and the Bloch-Kato exponential map, J. Inst. Math. Jussieu 10 (2011), 149-190.

[HKN] A. Huber, G. Kings, and N. Naumann, Some complements to the Lazard isomorphism, Compos. Math. 147 (2011), 235-262.

[L] M. Lazard, Groupes analytiques p-adiques, Publ. Math. Inst. Hautes Études Sci. 26 (1965).

[M] J. S. Milne, Arithmetic Duality Theorems, Perspect. Math. 1, Academic Press, Boston, 1986.

[N] N. Naumann, Arithmetically defined dense subgroups of Morava stabilizer groups, Compos. Math. 144 (2008), 247-270.

[O] T. Ono, On the Tamagawa number of algebraic tori, Ann. of Math. (2) 78 (1963), $47-73$.

[PR] V. Platonov and A. Rapinchuk, Algebraic Groups and Number Theory, Pure Appl. Math. 139, Academic Press, Boston, 1994.

[S] J.-P. Serre, Lie Algebras and Lie Groups, Harvard University Lectures, W. A. Benjamin, New York, 1965.

[W] A. Weil, Adeles and Algebraic Groups, with appendices by M. Demazure and T. Ono, Progr. Math. 23, Birkhäuser, Boston, 1982.

\author{
Annette Huber \\ Mathematisches Institut \\ Universität Freiburg \\ 9102 Freiburg \\ Germany \\ annette.huber@math.uni-freiburg.de \\ Guido Kings \\ Fakultät für Mathematik \\ Universität Regensburg \\ 93040 Regensburg \\ Germany \\ guido.kings@mathematik . uni-regensburg. de
}

